\title{
Beyond targeted searches: the need for system-level approaches to understanding the connection between astrochemistry and the emergence of life
}

Primary Author:

Mark A. Ditzler, NASA Ames Research Center,mark.ditzler@nasa.gov, (650)-604-1058.

Co-Authors:

Andro C. Rios, Blue Marble Space Institute of Science

Michel Nuevo, Bay Area Environmental Research Institute

Milena Popovic, Blue Marble Space Institute of Science

Rocco Mancinelli, Bay Area Environmental Research Institute

David Summers, SETI Institute

Jared T. Broddrick, NASA Ames Research Center

Lynn J. Rothschild, NASA Ames Research Center

Arthur L. Weber, SETI Institute 


\section{Summary of recommendations}

- Analyses of extraterrestrial materials should focus on gaining a system-level understanding of the dynamic processes that give rise to their organic inventories.

- Support should be increased for laboratory studies aimed at understanding the distinction between strictly abiotic chemical processes that do not lead to life and chemical processes that can lead to the emergence and proliferation of life.

Introduction. Over the past several decades astrochemists have identified a large inventory of abiotically synthesized chemical compounds that are also present in life on Earth. For this, they have analyzed meteorites and interplanetary dust particles (IDPs), analyzed cometary particles returned by the NASA Stardust mission, and simulated the chemical synthesis of these compounds (both experimentally and computationally) under abiotic conditions in environments ranging from interstellar dense clouds to the early Earth. Moreover, in the upcoming years, samples from asteroids Ryugu and Bennu will be returned by the JAXA Hayabusa 2 and NASA OSIRIS-REx missions, respectively, so they can be analyzed with all the techniques available in laboratories worldwide. We now have extensive inventories of abiotically generated organic compounds, from many environments, that include molecules essential to all living systems. However, the molecules that comprise and sustain life on Earth are likely not identical to either those that can give rise to life or those that support life elsewhere. Without a better understanding of the range of processes that can lead to the emergence of life in a variety of habitable environments, these inventories cannot be effectively leveraged in the search for life and their astrobiological significance will remain unknown.

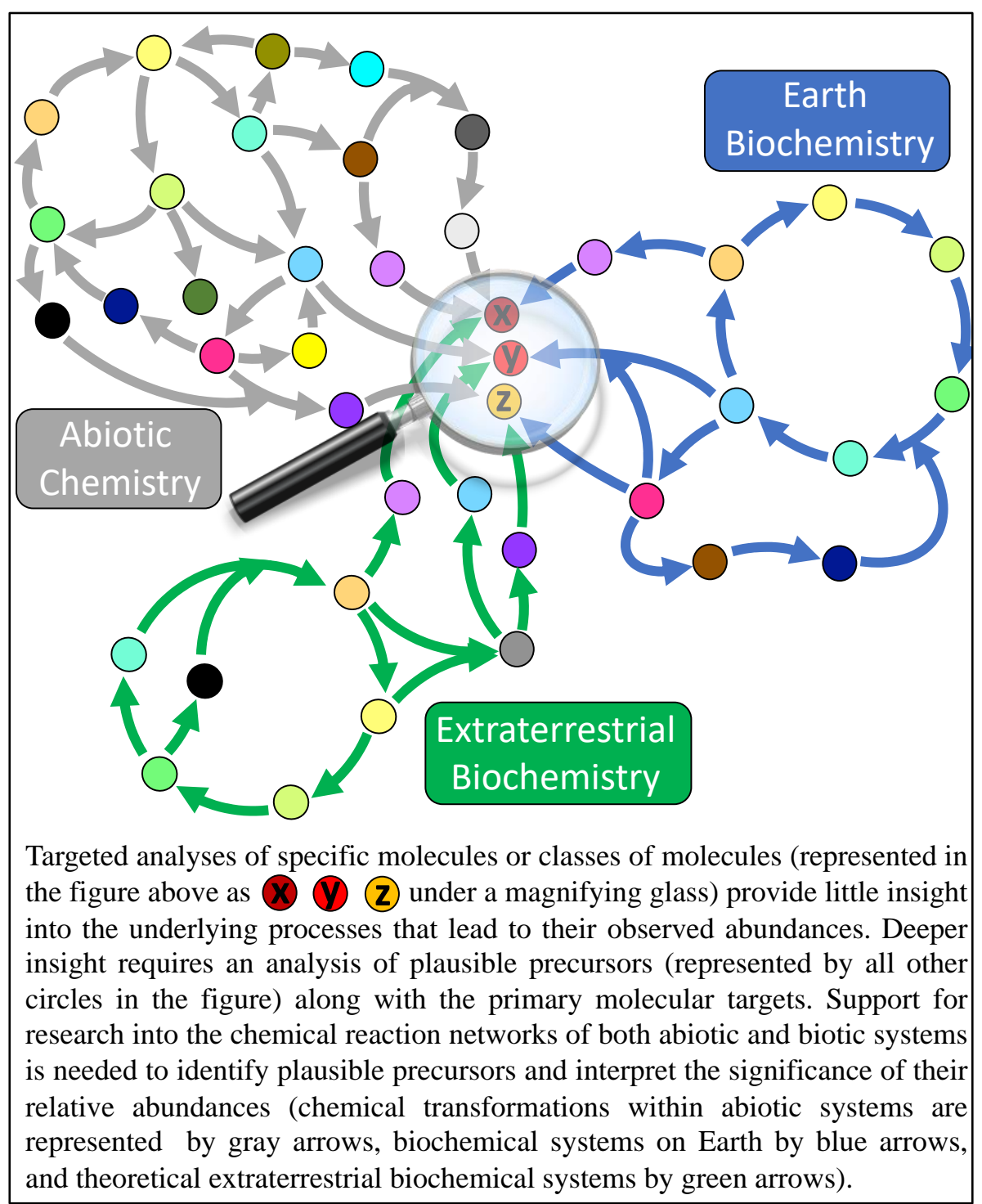


What are the "building blocks" of life? Living systems are characterized by modularity, with all known life transforming and recombining a shared set of molecular subunits to support its basic functions. The identity of these subunits is known for life on Earth and they can reasonably be defined as the "building blocks" of life, but this definition only includes building blocks used by living systems to sustain life on Earth. From an astrobiological perspective, this definition is incomplete. In astrobiology, we must concern ourselves with both the origin of life and alternative forms of life that may have arisen on other planets. We must therefore consider the building blocks used in the construction of life from non-living systems, and the range of all building blocks that may subsequently arise to sustain life in a range of planetary environments. Similar to extant life, the emergence of life from abiotic precursors likely relies on recombination of modular molecular building blocks, but the identity of these building blocks is far more difficult to determine. Seeking the connection between the initial building blocks that can lead to the emergence of life and the building blocks that can later sustain life should be the focus of future astrobiology missions.

Putting astrobiological molecular targets into context. Neither abiotic organic molecules nor bioorganic molecules should be considered in isolation, they are the products of dynamic chemical systems and their significance can only be understood within the context of those systems. Life on Earth evolved the ability to specifically transform simple chemical precursors into complex chemically related products. Abiotic processes generate many of the same products as biotic processes, but they are formed through different mechanisms. If independent forms of life exist, they likely evolved to produce a suite of products that also have at least some overlap with the products of life on Earth, but even in cases where end products are the same, the metabolic pathways could certainly be very different. In the following paragraphs we provide just a few examples that demonstrate why including an analysis of plausible precursors and products for any target molecule, or target category of molecules, is important for future astrobiology missions.

Amino acids: Amino acids were the first class of prominent biologically relevant organics detected in meteorites (Pizzarello et al. 2006). This included a large number (up to 70) and diversity of amino acids $(\alpha-, \beta-, \gamma-, \delta$-, etc.) (Shock $\&$ Schulte 1990). Furthermore, a wide range of amino acids were also detected in laboratory residues produced from the ultraviolet (UV) irradiation of astrophysical ices analogs under simulated astrophysical conditions (Bernstein et al. 2002; Muñoz Caro et al. 2002). Both meteoritic and laboratory investigations indicate that amino acid synthesis is a fairly common abiotic process in astrophysical environments.

In living cells, the synthesis and breakdown of many $\alpha$-amino acids ultimately centers on the connection to $\alpha$-keto acids that, in turn, fuel the tricarboxylic acid (TCA) cycle. Biological amino acids are used to construct proteins via peptide polymerization reactions, but searches for even simple polypeptides in meteorites have so far been inconclusive, highlighting a long-standing problem in connecting abiotic chemistry to complex functional products characteristic of known life. Namely, while peptide bond formation is a fairly simple polymerization reaction, driving this chemistry abiotically requires specific environmental conditions and/or chemical activation.

Although biochemistry on Earth is based on $\alpha$-amino acids linked to $\alpha$-keto acid precursors, it is entirely plausible that other forms of life evolved to metabolize $\alpha$-amino acids from different synthetic pathway precursors. One intriguing scenario takes advantage of the Strecker synthesis, a reaction that utilizes aldehydes, hydrogen cyanide, and ammonia for the production of $\alpha$-amino acids. In this reaction, when the abundance of ammonia is low, $\alpha$-hydroxy acid by-products become more prominent. Over time, the hydroxy and amino acids that are generated can serve as the ingredients needed to promote polypeptide synthesis. Hydroxy acids have been observed to act 
as chemical activators through a facile polymerization reaction achieved by ester bond formation (Forsythe et al. 2015). The thermodynamic instability of these ester bonds spontaneously promotes an ester/amide exchange by incorporating surrounding amino acids. This process leads to an intermediary hybrid polymer with a mixed ester and amide backbone known as a depsipeptide. Continued ester bond removal by amino acid substitution is what ultimately drives the completion of polypeptide synthesis. An extraterrestrial metabolism based on the aforementioned Strecker pathway might rely on depsipeptide intermediates for its protein synthesis.

Experimental work to explore alternative synthetic pathways, like the example given above, can heighten the sophistication of even simple analyses in life detection searches. The detection of elevated abundances of amino acids alone does little to reveal the processes that lead to those abundances and those abundances alone would serve as a poor biosignature. It is critical to understand the chemical system in which the amino acids exist. Identifying distinct synthetic precursors along with amino acids would help determine whether biological processes played a role and, if so, how those processes compare to the known biochemistry of Earth.

Organic acids: A broad class of compounds that contain one or more carboxylic acid groups, often termed organic acids, have a well-documented and widespread occurrence in meteorites (Pizzarello et al. 2006). For the chemically robust mono-, di-, and even tricarboxylic acids, the diversity of chemical structures often found within these classes reflect common abiotic synthetic processes. The presence of extraterrestrial $\alpha$ - and $\beta$-keto acids (Cooper et al. 2011) presents a particularly intriguing observation, given their notorious chemical instability (Cooper et al. 1983; Wolfenden 2011). It remains a matter of on-going investigation as to how such species are able to survive for long periods of time, while enduring meteoritic parent body alteration processes.

Organic acids associated with biology prominently occur as intermediary metabolites in the TCA cycle and are generated from catabolic pathways of amino acids, fatty acids, sugars, etc. Here the TCA cycle relies on these intermediary metabolites to fuel the majority of energetic and biosynthetic demands associated with primary metabolism. Whether life originated using a primitive metabolism based on the same TCA cycle organics or not is a matter of active investigation within the origin of life community.

If metabolic cycles are indeed a fundamental necessity for life, the TCA cycle would not necessarily be the only possibility. It has been pointed out that the selected intermediary metabolites do not appear to exhibit any special capabilities over other analogous compounds (Zubarev et al. 2015; Meringer \& Cleaves 2017). While the search for evidence of an extraterrestrial metabolism might encompass a much wider selection of organic acids, particular attention should still be given to finding the presence of chemically labile compounds that could be exploited by an organism as a source of energetic and biosynthetic utility. The $\alpha$ - and $\beta$-keto acids fulfill this role in biochemistry on Earth, and perhaps other water-based worlds, but for an organism in an ammonia-rich environment, analogous $\alpha$ - and $\beta$-imino acids would be more chemically relevant as metabolic intermediates.

Sugars and derivatives: Sugars and their derivatives sugar alcohols, sugar acids, sugar diacids, and their corresponding deoxy variants, have also been shown to be produced from the energetic processing of simple astrophysical ice analogs under abiotic conditions in laboratory experiments simulating cold astrophysical environments (Meinert et al. 2016; Nuevo et al. 2018). Sugar alcohols, sugar acids, sugar diacids, and several deoxysugar acids have been detected in abundance in carbonaceous meteorites (Cooper et al. 2001), but only a few sugars such as ribose and its isomers have recently been reported in small abundances (Furukawa et al. 2019). 
Sugars are involved in a wide range of foundational roles in modern biology. Six-carbon sugars, notably glucose, provide the majority of the carbon biomass, and, via the glycolytic pathway, serves as the ultimate fuel source for cells. Glucose is also used as the building block for cellulose, a structural polysaccharide for many organisms, which is also the single most abundant organic polymer on Earth. Other prominent sugars include ribose, a key structural component of both nucleic acids and the bioenergetic compounds ATP, NADH, FADH, etc. The widely differing functions of sugars found in biochemistry have long suggested to prebiotic chemists that sugars were vital to the emergence of life (Weber 1997, 1999). The well-known formose reaction, which leads to the autocatalytic generation of all sugars (Butlerow 1861; Breslow 1959), has also been viewed as a prebiotically plausible route for these compounds on the early Earth.

Like amino acids, sugars are chiral molecules, and while the formose reaction can generate sugars, these sugars are produced in racemic proportions. Homochirality is thought to be a fundamental property of life, and it still remains one of the unsolved problems in the origin of life research community, namely, could there have been a mechanism for enantioselective syntheses that gave rise to homochiral polymers? The detection of significant enantiomeric excesses of meteoritic sugar acids (Cooper \& Rios 2016) and amino acids (Cronin \& Pizzarello 1997; Glavin et al. 2020) provide evidence that pathways of abiotic, asymmetric syntheses of classes of biomolecules did occur early in our Solar System, even if the mechanism is still unknown. Life on other worlds would not necessarily be based on the same homochirality as on Earth, but molecular asymmetry is likely an important property for biomolecular functions.

Nucleobases, nucleosides, and nucleotides: Nucleobases are common products of chemistry in astrophysical environments. Similar to amino acids, the purine and pyrimidine nucleobases found in meteorites (Martins et al. 2008; Callahan et al. 2011) and laboratory experiments (Nuevo et al. 2014; Materese et al. 2018; Oba et al. 2019) display a wider diversity than what is found in biology. Nucleotides are the building blocks of RNA and DNA, and among the most complex monomers found in biology, given their unique chemical composition and specific connectivity required to form nucleic acid polymers. Nucleotides are often described as being composed of three subunits that include a five-carbon sugar (ribose or deoxyribose), two types of nucleobase classes (purines and pyrimidines), and a simple inorganic phosphate. These components play only minor roles as isolated subunits in living systems, arising only as nucleotide degradation products. It is unclear what role, if any, these isolated subunits played in the emergence of life.

The presence of ribose and several pyrimidine and purine nucleobases in carbonaceous meteorites and in laboratory ice photochemistry residues is often interpreted in the context of these subunits acting as precursors to life. But despite nearly 40 years of laboratory studies, a plausible abiotic reaction that can link each of the canonical nucleobases to ribose with high yield and in a regiospecific manner has yet to be identified. This is further compounded by the lack of any regiospecific route to link the corresponding nucleotides without chemical activation. Nucleotide synthesis pathways that do not include the synthesis of nucleobase intermediates may be more likely routes to generating nucleotides along a path that can lead to the emergence of life. Success in generating nucleotides has been found from prebiotic reactions that, like the biological pathways, synthesize the nucleobase directly on the ribose moiety (Powner et al. 2009).

Nucleic acids may ultimately be a product of biochemical evolution on Earth, but if independent forms of life did emerge with a similar genetic polymer, its components or anabolic/catabolic pathways could still be very different. It has been previously demonstrated that even simple substitutions using other nucleobases and sugar moieties resulted in genetically viable polymers (Benner 2004; Yu et al. 2012). These demonstrations are also viewed as possible 
evidence for how RNA could have evolved from a simpler prebiotic polymer (Hud et al. 2013). Even if extraterrestrial life is found to rely on the same genetic alphabet as RNA and DNA (A, T/U, C, G), it might have evolved with different biosynthetic precursors. As demonstrated by Powner et al. (2009), there are many synthetic precursors that could lead to the canonical nucleobases and are also ubiquitous in astrophysical environments.

Amphiphiles: Amphiphilic compounds (i.e., molecules consisting of both hydrophilic and hydrophobic parts), have been found in both carbonaceous meteorites (Deamer \& Pashley 1989) and laboratory experiments simulating chemical processes of simple ices $\left(\mathrm{H}_{2} \mathrm{O}, \mathrm{CH}_{3} \mathrm{OH}, \mathrm{NH}_{3}, \mathrm{CO}\right.$, $\mathrm{CO}_{2}$, etc.) in cold astrophysical environments (Dworkin et al. 2001). This class of molecules is of great astrobiological interest because in aqueous solution they can spontaneously self-assemble into vesicle-like structures that could have played a key role in the origin of life.

Although the spontaneous formation of vesicles in aqueous extracts of both meteoritic samples and laboratory ice photolysis residues clearly points to the presence of amphiphiles, it is not clear what the molecular structures of these compounds are, and whether the constituents of vesicles from these two sources are similar. Liquid chromatography separation of these residues indicated that the amphiphiles consist of several classes of compounds with amphiphilic properties rather than only one single class. These vesicles fluoresce under UV light, although it has not been demonstrated yet whether the fluorescence is caused by the amphiphiles themselves, or by other fluorescent molecules encapsulated inside the vesicles. Indeed, studies of ice photolysis residues that form vesicles in aqueous solutions have shown that dyes can be trapped inside these vesicles after successive wet/dry cycles (Dworkin et al 2001). Similar wet/dry cycles are a possible mechanism by which relatively impermeant molecules could be encapsulated into membranes of proto-cells in prebiotic environments.

Amphiphiles play a variety of roles in biology and are themselves highly diverse. Phospholipids, a major component of the cell membrane bilayer, are produced by combining either fatty acid or isoprene building blocks with glycerol-phosphate. Other amphiphiles, such as sterols, hopanoids, and proteins, tune the physical and biological properties of membranes. Biological amphiphiles are complex and the result of long evolutionary processes. Mixtures of amphiphiles of varying lengths are key to the formation of vesicle-like structures under a variety of conditions (Namani \& Deamer 2008; Maurer et al. 2018; Jordan et al. 2019). It is unlikely that abiotically synthesized amphiphilic compounds resemble the lipids used in modern biological membranes. Nonetheless, the presence of amphiphiles in abundance in meteorites and laboratory ice irradiation residues, combined with their ability to form vesicles under different conditions, suggests that they could have been precursors to the membranes of the protocells that allowed life to develop on the early Earth and perhaps other aqueous environments in our Solar System.

Polycyclic aromatic hydrocarbons (PAHs): PAHs are ubiquitous in astrophysical environments, and potentially constitute the most abundant form of organic carbon in the universe (Allamandola et al. 1989; Puget \& Léger 1989). PAHs present in these environments are believed to consist of 50-100 carbon atoms on average (Tielens 2020), but their size mostly depends on the radiation conditions, with larger PAHs present in lower radiation environments. Although PAHs do not play any direct role in known biological systems on Earth, they share structural and functional features with several biological compounds such as quinones (Suttie 1979; Thurl et al. 1985) and may have served as intermediates in protobiology's redox chemistry and were then subsequently replaced (Dalai \& Sahai 2019). The hydrophobic nature of these large aromatic systems may also provide 
the necessary chemical attributes to properly function for some of the most exotic lifeforms we might encounter in, for example, a hydrocarbon-rich environment like the surface of Titan.

Insoluble macromolecular organic material (IMOM): IMOM represents the large majority of the organic carbon present in meteorites and IDPs (Alexander et al. 2017; Glavin et al. 2018) and is believed to preserve information about the abiotic processes that took place in the early Solar System and even in the presolar nebula. On Earth, IMOM preserves the history of biology in the form of kerogen (Vandenbroucke \& Largeau 2007). Even in our highly active biosphere, the majority of organic carbon on Earth is currently incorporated into IMOM.

IMOM has structural features that are diagnostic of its origins. Terrestrial IMOM contains structural elements that are inherited from the parent biomolecules. Examples include $n$-alkanes derived from fatty acids (van Kaam-Peters et al. 1997; Love et al. 1998; Duda et al. 2018), isoprene chains from pigments and Archaeal lipids, hopane and sterane units from bacterial and eukaryal lipids, respectively (van Kaam-Peters et al. 1997; Love et al. 1998; Sugden et al. 2002), and porphyrin structures derived from chlorophylls and hemes (Sundararaman et al. 1988; Huseby et al. 1996). IDP and meteoritic IMOMs contain small aromatic units linked by short and highly branched aliphatic chains (Cody \& Alexander 2005; Remusat et al. 2005; Huang et al. 2007). The high abundance and long-term preservation of these materials makes a detailed characterization of IMOM, together with its formation and transformation, essential to any meaningful understanding of organic molecules in both biotic and abiotic chemical systems.

Putting it all together. We have highlighted several classes of organic molecules, their presence in abiotic environments, their biotic characteristics as they exist on Earth, and their potential role in extraterrestrial biochemistries. It is clear that the building blocks of life can have various abiotic origins and are bound to permeate our Solar System and other planetary systems in our galaxy. This should be expected, as abiotic chemistry must have provided the feedstock for prebiotic chemical evolution that ultimately gave rise to the origin of life at some point in the early history of Earth. Although abiotic and biotic chemistries have commonalities at the level of individual building blocks, research endeavors from the origin of life field and knowledge of extant biochemistry can guide system-level analyses that differentiate between extraterrestrial biotic and abiotic chemistry. While abiotic chemistry can exhibit multiple pathways towards a particular compound, biotic systems evolve chemical systems that capitalized on a distinct subset of precursors among the abiotic possibilities. In the last decade, new approaches have emerged to understand complex networks of interacting molecules in chemical and biological systems. These approaches, which form the basis of systems chemistry and systems biology, should be incorporated into astrobiology missions by including analyses of potential precursors associated with any molecular target. In support of these analyses, significant support for research in prebiotic chemistry will be needed to enhance our knowledge of abiotic synthetic routes to the building blocks of life and to uncover possible pathways that other forms of life might have adopted.

\section{References:}

Alexander, C. M. O’D., et al. (2017) Geochem., 77, 227-256.

Allamandola, L. J., et al. (1989) Astrophys. J. Suppl. Ser., 71, 733-755.

Bernstein, M. P., et al. (2002) Nature, 416, 401-403.

Benner, S. A. (2004) Acc. Chem. Res., 37, 784-797.

Butlerow, A. (1861) Justus Liebigs Ann. Chem., 120, 295-298.

Breslow, R. (1959) Tetrahedron Lett., 21, 22-26.

Callahan, M. P., et al. (2011) Proc. Natl. Acad. Sci., 108, 13995-13998. 
Cody, G. D. \& Alexander, C. M. O’D. (2005) Geochim. Cosmochim. Acta, 69, 1085-1097.

Cooper, A. J. L., et al. (1983) Chem. Rev., 83, 321-358.

Cooper, G. \& Rios, A. C. (2016) Proc. Natl. Acad. Sci., 113, E3322-E3331.

Cooper, G., et al. (2001) Nature, 414, 879-883.

Cooper, G., et al. (2011) Proc. Natl. Acad. Sci., 108, 14015-14020.

Cronin, J. R. \& Pizzarello, S. (1997) Science, 275, 951-955.

Dalai, P. \& Sahai, N. (2019) J. Phys. Chem. C, 124, 1469-1477.

Deamer, D. W. \& Pashley, R. M. (1989) Orig. Life Evol. Biosph., 19, 21-38.

Duda, J.-P., et al. (2018) Biogeosci., 15, 1535-1548.

Dworkin, J. P., et al. (2001) Proc. Natl. Acad. Sci., 98, 815-819.

Forsythe, J. G., et al. (2015) Angew. Chem. Int. Ed., 54, 9871-9875.

Furukawa, Y., et al. (2019) Proc. Natl. Acad. Sci., 116, 24440-24445.

Glavin, D., et al. (2018) In Primitive meteorites and asteroids, pp. 205-271.

Glavin, D., et al. (2020) Chem. Rev., 120, 4660-4689.

Hud, N., et al. (2013) Chem. Biol., 20, 466-474.

Huang, Y., et al. (2007) Earth Planet. Sci. Lett., 259, 517-525.

Huseby, B., et al. (1996) Org. Geochem., 24, 691-703.

Jordan, S. F., et al. (2019) Nat. Ecol. Evol., 3, 1705-1714.

Love, G. D., et al. (1998) Org. Geochem., 28, 797-811.

Martins, Z., et al. (2008) Earth Planet. Sci. Lett., 270, 130-136.

Materese, C. K., et al. (2018) Astrophys. J., 864, 44.

Maurer, S. E., et al. (2018) Langmuir, 34, 15560-15668.

Meinert, C., et al. (2016) Science, 352, 208-212.

Meringer, M. \& Cleaves, H. J. (II) (2017) Sci. Rep. 7, 17540.

Muñoz Caro, G. M., et al. (2002) Nature, 416, 403-406.

Namani, T. \& Deamer, D. W. (2008) Orig. Life Evol. Biosph., 38, 329-341.

Nuevo, M., et al. (2014) Astrophys. J., 793, 125.

Nuevo, M., et al. (2018) Nat. Commun., 9, 5276.

Oba, Y., et al. (2019) Nat. Commun., 10, 4413.

Pizzarello, S., et al. (2006) In Meteorites and the Early Solar System II, pp. 625-651.

Puget, J.-L. \& Léger, A. (1989) Annu. Rev. Astron. Astrophys., 27, 161-198.

Powner, M. W., et al. (2009) Nature, 459, 239-242.

Remusat, L., et al. (2005) Geochim. Cosmochim. Acta, 69, 4377-4386.

Shock, E. L. \& Schulte, M. D. (1990) Geochim. Cosmochim. Acta, 54, 3159-3173.

Sugden, M. A. \& Abbott, G. D. (2002) Org. Geochem., 33, 1515-1521.

Sundararaman, P., et al. (1988) Geochim. Cosmochim. Acta, 52, 2337-2341.

Suttie, J. W. (1979) In Vitamin K metabolism and vitamin $K$ dependent proteins.

Thurl, S., et al. (1985) Biol. Chem. Hoppe-Seyler, 366, 1079-1083.

Tielens, A. G. G. M. (2020) In Molecular Astrophysics.

Vandenbroucke, M. \& Largeau, C. (2007) Org. Geochem., 38, 719-833.

van Kaam-Peters, H. M. E., et al. (1997) Org. Geochem., 27, 399-422.

Weber, A. L. (1997) J. Mol. Evol., 44, 354-360.

Weber, A. L. (1999) Orig. Life Evol. Biosph., 30, 33-43.

Wolfenden, R. (2011) Ann. Rev. Biochem., 80, 645-667.

Yu, H., et al. (2012) Nat. Chem., 4, 183-187.

Zubarev, D. Y., et al. (2015) Sci. Rep., 5, 8009. 